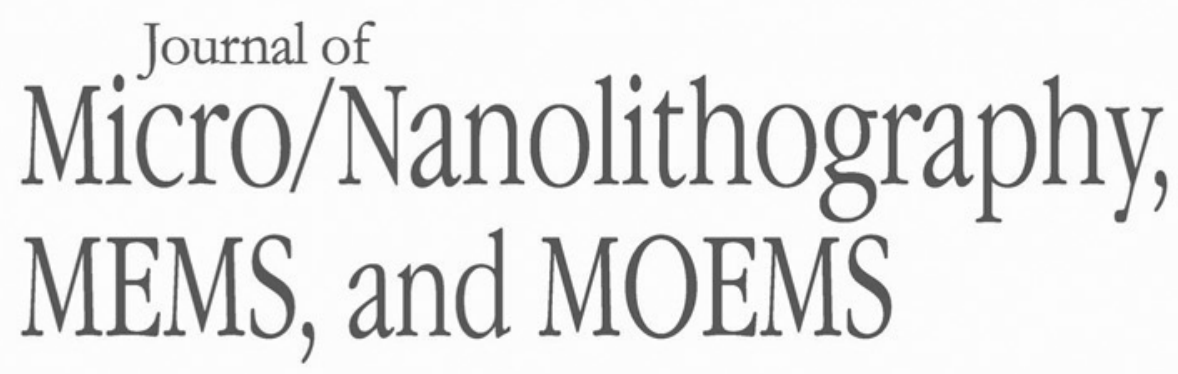

Nanolithography.SPIEDigitalLibrary.org

\title{
Fabrication and analysis of metallic nanoslit structures: advancements in the nanomasking method
}

Stephen J. Bauman

Ahmad A. Darweesh

Desalegn T. Debu

Joseph B. Herzog 


\title{
Fabrication and analysis of metallic nanoslit structures: advancements in the nanomasking method
}

\author{
Stephen J. Bauman, ${ }^{a}$ Ahmad A. Darweesh, ${ }^{a}$ Desalegn T. Debu, ${ }^{b}$ and Joseph B. Herzog ${ }^{a, b, *}$ \\ aUniversity of Arkansas, Microelectronics-Photonics Graduate Program, Fayetteville, Arkansas, United States \\ bUniversity of Arkansas, Department of Physics, Fayetteville, Arkansas, United States
}

\begin{abstract}
This work advances the fabrication capabilities of a two-step lithography technique known as nanomasking for patterning metallic nanoslit (nanogap) structures with sub-10-nm resolution, below the limit of the lithography tools used during the process. Control over structure and slit geometry is a key component of the reported method, exhibiting the control of lithographic methods while adding the potential for mass-production scale patterning speed during the secondary step of the process. The unique process allows for fabrication of interesting geometric combinations such as dual-width gratings that are otherwise difficult to create with the nanoscale resolution required for applications, such as nanoscale optics (plasmonics) and electronics. The method is advanced by introducing a bimetallic fabrication design concept and by demonstrating blanket nanomasking. Here, the need for the secondary lithography step is eliminated improving the mass-production capabilities of the technique. Analysis of the gap width and edge roughness is reported, with the average slit width measured at $7.4 \pm 2.2 \mathrm{~nm}$. It was found that while no long-range correlation exists between the roughness of either gap edge, and there are ranges in the order of tens of nanometers over which the slit edge roughness is correlated or anticorrelated across the gap. This work helps quantify the nanomasking process, which aids in future fabrications and leads toward the development of more accurate computational models for the optical and electrical properties of fabricated devices. $\odot$ The Authors. Published by SPIE under a Creative Commons Attribution 3.0 Unported License. Distribution or reproduction of this work in whole or in part requires full attribution of the original publication, including its DOI. [DOI: 10 .1117/1.JMM.17.1.013501]
\end{abstract}

Keywords: nanofabrication; nanogap; nanoslit; sub-10 nm.

Paper 17132P received Oct. 13, 2017; accepted for publication Dec. 7, 2017; published online Jan. 2, 2018.

\section{Introduction}

The ability to create metallic nanostructures, whether via top-down or bottom-up methods, has become increasingly common, if not necessary, for many areas of modern technological development. Advances in self-assembling chemical processes, lithographic methods involving accelerating ions and particles, and high-precision deposition and etch techniques have enabled much of this fabrication progress. ${ }^{1-5}$ These advances will enable new and exciting technologies that take advantage of properties, such as increased surface area at the nanoscale, high-density arrays of structures, and even Angstrom-scale features or gaps (also referred to as slits) among structures. ${ }^{6-9}$

High-precision nanofabrication may produce a wide range of structures that are beneficial for their chemical, mechanical, electrical, optical, or combined properties across nearly endless applications. ${ }^{10-14}$ Plasmonic nanostructures, which interact with light in unique ways depending on the surrounding materials and device geometry, have been applied to improved electronics performance and enhancement of optical signals. ${ }^{15-18}$ In addition to nanostructures producing electric field enhancement, slits among structures (specifically those approaching $10 \mathrm{~nm}$ and smaller) ${ }^{19}$ have been shown to further increase the local field strength. ${ }^{20-24}$

One limitation of using nanoslits for this type of plasmonic enhancement in various applications is the difficulty of reliable fabrication at the sub-10-nm scale, which is below

*Address all correspondence to: Joseph B. Herzog, E-mail: jbherzog @ uark.edu the resolution limit of most lithography systems, and over large wafer-scale areas. ${ }^{25}$ Existing gap-fabrication methods, such as focused ion beam (FIB) milling, ${ }^{26-28}$ electromigration, ${ }^{29-33}$ mechanical break junctions, ${ }^{34}$ or photo/electron/ ion beam lithographies, ${ }^{26-28,35,36}$ have the limitation that they must create gaps serially. It is crucial to consider the time of fabrication for a large area of nanostructures or slits when considering scaling up of the technology for applications beyond pure basic research. A variety of self-assembly techniques have emerged as promising candidates for large-area fabrication of high densities of nanogaps, some even with control over gap sizes. ${ }^{37-44}$ The ideal fabrication technique would perfectly couple the dimensional control of methods such as lithography with the rapid and large-scale, simultaneous creation of the desired nanostructures and nanoslits.

Various techniques make use of a sacrificial layer or layers of material during top-down lithographic processes to improve the resolution or other properties of the fabrication. Even during its advent two hundred years ago, the basic concept of lithography (from the Greek lithos, "stone" and graphien, "to write") relied on a sacrificial resist layer. ${ }^{11}$ Resists sensitive to exposure via an electron beam allow the user to take advantage of the smaller wavelength, and therefore, higher diffraction-limited resolution of accelerated electrons over the ultraviolet light used with photosensitive resists. Sacrificial layers made from metals, semiconductor oxides, or other materials used during top-down lithography processes may provide additional benefits due to specific material properties not present in lithographic resists, which are often organic compounds. One benefit of using 
different materials is the ability to take advantage of different etchants or solvents that react solely with the desired layer material. The sacrificial layer may simply protect another surface from contamination, ${ }^{45}$ fogging, ${ }^{46}$ or other nanoscale defects, or it may be implemented to remove these defects from the important surface. ${ }^{47}$ Some imprint-type techniques rely on a sacrificial material as a carrier from which the desired structures are removed after processing or this layer may provide structure to a specific geometric design during fabrication. ${ }^{48-50}$

The addition of a sacrificial aluminum layer prior to FIB milling has been demonstrated to improve the resolution and edge smoothness in a process called metal-assisted FIB. ${ }^{51}$ Here, the sacrificial metal layer works to protect the working material from ion-induced damage and redeposition of milled working material. This technique was used in the production of improved templates for nanoimprint lithography and two-dimensional plasmonic open-ring nanostructure arrays with significantly improved absorption due to increased structural integrity of the patterns. While this and other sacrificial masking techniques provide the mentioned benefits, they do not necessarily directly improve the resolution of the corresponding nanofabrication process.

This work describes the nanomasking technique, which is an advanced fabrication method that takes advantage of a unique lithography and deposition process to create nanoslits adjacent to metallic nanostructures to produce nanoscale devices. As we have outlined previously, preliminary results have demonstrated the ability to simultaneously fabricate sub-10-nm slits with a density of over 500 million per square $\mathrm{cm}^{52}$ Nanomasking has also been used to simultaneously create gaps in two dimensions, which is not possible with some serial techniques. An additional benefit of nanomasking is that it has been shown to be capable of simultaneously fabricating both sub-10-nm slits and adjacent sub-20-nm metallic structures. ${ }^{52}$ The nanomasking technique overcomes this limitation via a unique multistep lithography process to simultaneously produce many sub-10-nm gaps across a surface. The geometrical control of this technique has been demonstrated as well, in which gaps can be created adjacent to sublithography-limited structures with control over their shapes and sizes. The increased geometric control over nanoscale structures and slits has resulted in the patterning of metallic devices that can be applied to developments in fields, such as plasmonics, nanoscale and nonlinear optics, photonic crystals, waveguides, electronics, and microfluidics. ${ }^{53-62}$ This work improves the nanomasking technique beyond the previous results by introducing a bimetallic nanoslit design and demonstrates blanket nanomasking, which improves upon the original technique by eliminating one of the lithography steps. Here, we also carefully analyze and quantify the gap wall roughness and sidewall correlation, which reveal important insights for device design and applications.

\section{Nanomasking Fabrication}

In Fig. 1, we introduce a capability of the nanomasking technique. Figure 1(a) shows the standard technique, while Fig. 1(b) shows an additional capability of this technique: bimetallic nanoslit fabrication. This advanced method of fabricating two different metals with nanoslit spacing is an innovative concept and has potential for interesting optoelectronic applications. ${ }^{63}$

The standard nanomasking process utilizes a two-step lithography process to obtain sub-10-nm gaps with a high degree of control over structure geometries. Figure 1 outlines the key steps in the process for creating (a) an Au grid structure and (b) concentric circular patterns utilizing two

\section{(a)}



(iii)

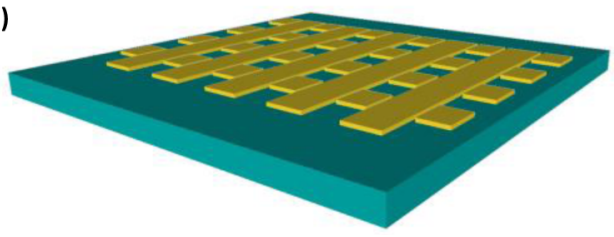

(b)

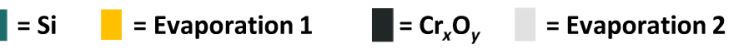

(i)

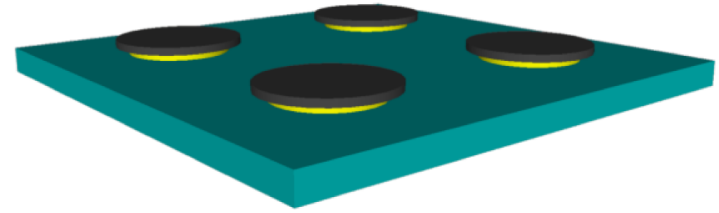

(ii)



(iii)

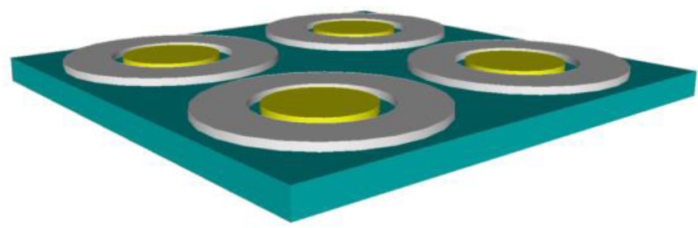

Fig. 1 3-D sketch of the nanomasking technique for (a) a grid pattern (two Au evaporations shown) and (b) bimetallic circular patterns (two evaporation metals shown): (i) result of the first lithography step and deposition showing structures of $\mathrm{Au}$ topped with $\mathrm{Cr}_{x} \mathrm{O}_{y}$, (ii) result of the second lithography step and deposition showing secondary metallic structures masked by $\mathrm{Cr}_{x} \mathrm{O}_{y}$, and (iii) result of etching $\mathrm{Cr}_{x} \mathrm{O}_{y}$ showing metallic geometries as designed with nanogaps among adjacent structures. 
different evaporation materials. ${ }^{63}$ After the primary structures have been patterned via standard electron beam lithography (EBL) or photolithography, resist development, and evaporation, the first step of the nanomasking process takes place as shown in Fig. 1(i). During the evaporation step of (i), another material layer is evaporated atop the desired material for the primary structures; this layer is, crucially, a metal or other material that will undergo oxidation and expansion under ambient or controlled conditions. In our work and, therefore, the sketches shown in Fig. 1, a $\mathrm{Cr}$ layer is used to create this nanoscale mask layer upon oxidation. The overhanging oxidized layer acts to shield the substrate from further material evaporation during the second lithography, development, and evaporation step, as shown in Fig. 1(ii). The second important criterion for choosing a nanomask layer material is that it must be etchable without damaging the substrate, any necessary adhesion layer, or the other desired primary and secondary evaporation materials. Thus, upon etching the nanomask layer, the resulting patterns consist of the primary and secondary materials separated by nanogaps where they overlap as designed, with the nanogap size being tunable by controlling the oxidation of the mask material. Example of final structures is shown in Fig. 1(iii).

Not shown in Fig. 1 but important for fabrication of $\mathrm{Au}$ structures on a $\mathrm{Si} / \mathrm{SiO}_{2}$ substrate is a $\mathrm{Ti}$ adhesion layer. In the work described here, this was typically 1.0 to $1.5 \mathrm{~nm}$ of $\mathrm{Ti}$, with $15 \mathrm{~nm}$ of $\mathrm{Au}$ as both the primary and secondary evaporation material. Previous work studying the effects of a Ti adhesion layer on the plasmonic response of an Au nanostructure to incident light illumination has shown that the optical enhancement produced by the structures decreases with increasing Ti layer thickness. ${ }^{64-68}$ Therefore, for optical applications, ideally no adhesion layer would be used, but in the case in which it is required, the smallest possible adhesion layer should be used to preserve the optical characteristics of the patterned structures. The thickness of the $\mathrm{Au}$ layer has also been found to significantly affect the optical response of a patterned nanostructure, with and without nanoslits. ${ }^{69-73}$ Beyond consideration of the final application, however, these thicknesses should be chosen such that the nanomasking effect can still pattern the desired nanostructures/slits. The thickness of the $\mathrm{Cr}$ layer is critical if tight control over the gap width is desired, as the thickness affects the lateral expansion of Cr oxidation that occurs upon exposure to oxygen, affecting the gap width as reported in work by Fursina et al. ${ }^{74}$

Nanomasking has successfully demonstrated the fabrication of $\mathrm{Au}$ grid structures on a $\mathrm{Si} / \mathrm{SiO}_{2}$ substrate as designed and as outlined in Fig. 1. Scanning electron microscopy (SEM) images were taken of the resulting structures, as shown colorized in Fig. 2. Different design widths and spacings of the primary nanowires were found to produce different widths of the adjacent secondary Au structures. With the designs producing a resulting primary width of $165 \mathrm{~nm}$, secondary structures were measured to be $65 \mathrm{~nm}$ as shown in Figs. 2(a) and 2(b). Increasing the primary width to $200 \mathrm{~nm}$ resulted in a secondary structure width of $40 \mathrm{~nm}$ [Fig. 2(c)].

The fact that adjusting the primary $\mathrm{Au}$ structure width demonstrates the tunability of the resulting grids via the nanomasking technique. This will prove useful in future experiments by allowing designers to strategically pattern geometries that show optimal enhancement in simulations. This will accelerate the learning process in determining the efficiency of the grid structures as SERS substrates, for example.

The ability of the nanomasking process to fabricate nanogaps has been demonstrated as a proof of concept for the possible large-scale integration of the technique. This work also demonstrated the simultaneous fabrication of nanogaps and adjacent nanostructures that are both below the lithography resolution limit. This has been described for patterns aligned on the same center point as with the circle patterns shown in Fig. 1. Varying the overlap of the two patterns, however, has the effect of changing the width of the resulting secondary metal, creating an adjacent sublithography-limited structure separated via nanoslit.

Figure 3 shows colorized SEM images of the result of varying the overlap between a square primary pattern and a rectangular secondary pattern. One larger rectangular and one square pattern with different amounts of overlap are shown in Fig. 3(a). The higher magnification image
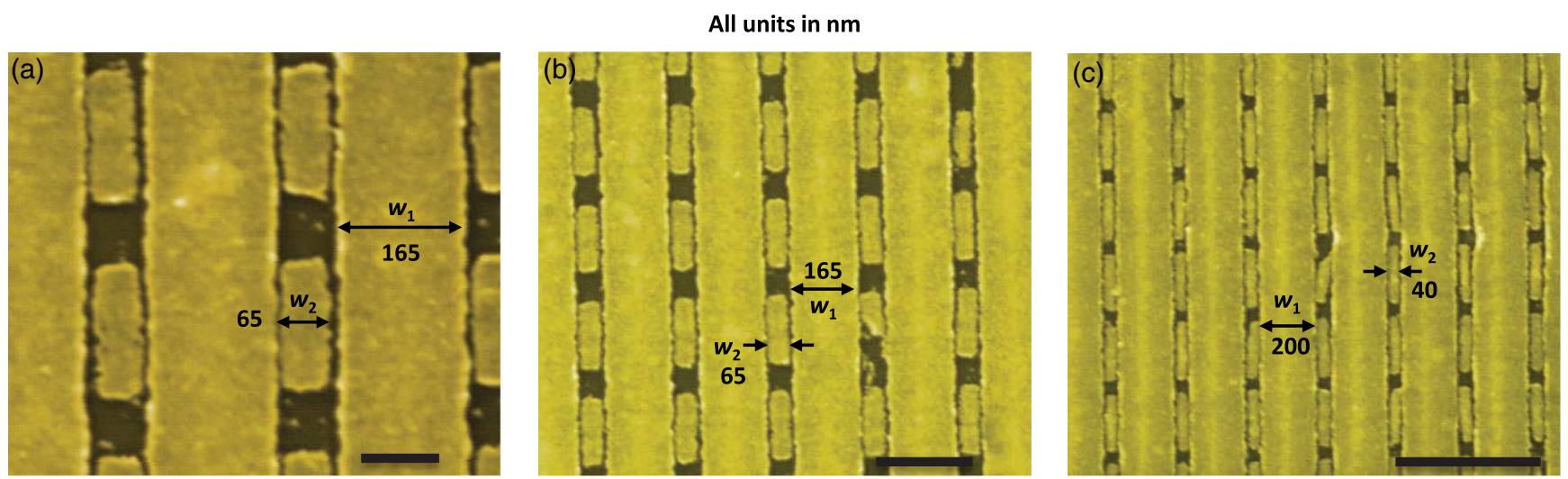

Fig. 2 SEM images of Au dual-width grid structures fabricated on a $\mathrm{Si} / \mathrm{SiO}_{2}$ substrate via nanomasking with different designed structure widths. The width, $w_{1}$, and spacing of the primary structures control the width of the adjacent wires, $w_{2}$. In (a) and (b), $w_{1}=165$ and $w_{2}=65 \mathrm{~nm}$, and in (c), $w_{1}=200 \mathrm{~nm}$ and $w_{2}=40 \mathrm{~nm}$. The structure width standard deviation was found to be $3.03 \mathrm{~nm}$. Scale bars in (a), (b), and (c) are 100,250 , and $500 \mathrm{~nm}$, respectively. 

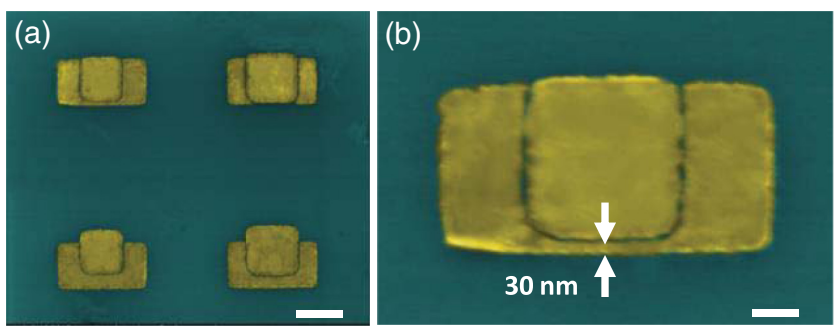

Fig. 3 Colorized SEM images of the results of nanomasking fabrication for a square primary pattern overlapping a secondary rectangular pattern: (a) results for two primary pattern sizes and two different degrees of overlap between primary and secondary patterns and (b) close-up of the top left structure in (a) showing the resulting nanostructure width. Scale bars in (a) and (b) are 500 and $100 \mathrm{~nm}$, respectively.

(b) shows one case in which the square pattern and rectangular pattern were overlapped so that structures are formed adjacent to the gap; below the square, a 30-nm metal nanostructure was formed with features below the typical lithography limit of the EBL system used in this work $(\sim 60 \mathrm{~nm})$. This highlights the capability to use the nanomasking technique to not only fabricate sub-10-nm slits but also as means to overcome lithography resolution limits for nanostructures.

Next, we show another advancement in the nanomasking technique, blanket nanomasking, which eliminates the need for the secondary lithography, reducing the process down to only one lithography step. This has the advantage of time and cost savings in the fabrication process. Here, it is used to create an array of parallel nanowires with different widths separated by nanogaps. The process shown in Fig. 4 begins, again, with patterning a desired geometry using lithography. This work used EBL, yet photolithography could be utilized instead for larger structures and rapid patterning. The desired metal and $\mathrm{Cr}$ layer were then deposited, and the $\mathrm{Cr}$ was allowed to oxidize (under ambient conditions in this work). This is shown in Fig. 4(a) with parallel Au nanowires that have an oxidized $\mathrm{Cr}$ layer overhanging the edge of the Au. From here, a second deposition of Au is all that is needed to produce more parallel nanowires, separated from the primary structures by nanogaps on both sides [Figs. 4(b) and 4(c)]. The deposition covers the entire sample area, and the $\mathrm{Cr}$ mask is still able to produce nanogaps adjacent to the wires. Figure 4(d) shows colorized SEM images of the results of this type of fabrication process. The width of the primary and secondary nanowires could be controlled in a design such as this to optimize the plasmonic response to specific wavelengths of incident light. ${ }^{9}$

Thus, blanket nanomasking has been shown to be capable of patterning nanogap structures over a large area without the need for a secondary lithography step. The geometrical design possibilities are limited compared to the standard nanomasking process, but blanket deposition over a sample containing nanostructures may prove useful for economical mass production of devices. We have shown that the optical response and plasmonic nature of dual-width nanogap gratings, as shown in Fig. 4(d), can be more beneficial than that of standard single-width structures for photodetector and spectroscopy enhancement applications. ${ }^{75,76}$ These works help to demonstrate the value of nanomasking fabrication, which allows for the dual-width grating structure with the added benefit of nanoslit separation. (a)

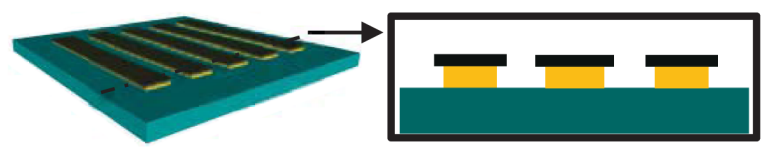

(b)



(c)

(d)
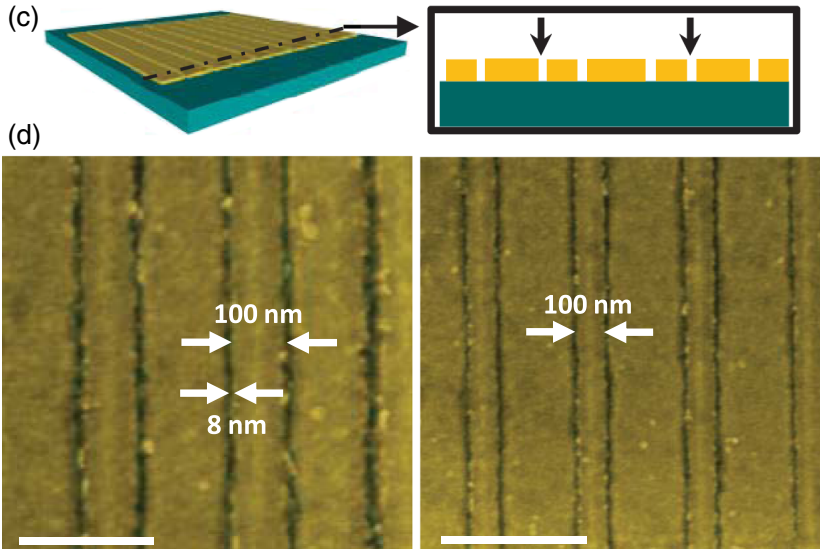

Fig. 4 Blanket nanomasking of parallel nanowires. 3-D sketches and cross-sectional views of (a) the result after the first lithography step, metal deposition (gold and gray), and Cr layer oxidation (gray), (b) the secondary metal deposition step, and (c) the resulting parallel nanowires separated by nanogaps. (d) Colorized SEM images of the resulting dual-width $\mathrm{Au}$ grid structure. Scale bars are 250 and $500 \mathrm{~nm}$, respectively, and the gap width is $\sim 8 \mathrm{~nm}$.

\section{Nanoslit Analysis}

Having demonstrated various geometrical fabrication capabilities via nanomasking, we then analyzed the structure of nanoslits created by the process. Three high-resolution SEM images of nanogaps (taken using an FEI Nova Nanolab 200) were studied to determine the gap widths along the length of the gap, as well as the correlation between the two gap edges in each case. The fabrication conditions for the studied patterns included deposition of a $1.5-\mathrm{nm} \mathrm{Ti}$ adhesion layer, $15 \mathrm{~nm}$ of $\mathrm{Au}$, a $1.5-\mathrm{nm} \mathrm{SiO}_{2}$ separation layer between the $\mathrm{Au}$ and $\mathrm{Cr}$, and $15 \mathrm{~nm}$ of $\mathrm{Cr}$ allowed to oxidize under ambient conditions. The gap widths were determined by measuring the full width at half maximum of the SEM image pixel values along a line drawn perpendicularly across the gap. The location of each edge point was determined in this manner along a gap length, $L$, of $100 \mathrm{~nm}$. From these edge locations, the gap width, average gap width, deviation from the mean, and edge correlation were calculated. The mean value across the three gaps was found to be $7.4 \mathrm{~nm}$ with a standard deviation of $2.2 \mathrm{~nm}$. The analyzed gaps are shown in the SEM images at the top of Fig. 5 with the specific locations for each gap measurement length, $L$, labeled. The red plots shown in the middle row of Fig. 5 display the gap width deviation from the mean, $\Delta g$, versus $L$. The red histogram at the right side of Fig. 5 plots the number of occurrences, $N$, for gap widths over different $\Delta g$ ranges.

The blue plots display the population correlation coefficient, $\rho$, versus $L$, with 1 representing complete correlation and -1 representing complete anticorrelation. From these data, it was found that there are ranges in the order of tens of nanometers along each gap over which the secondary structure edge is highly correlated with the edge position of 

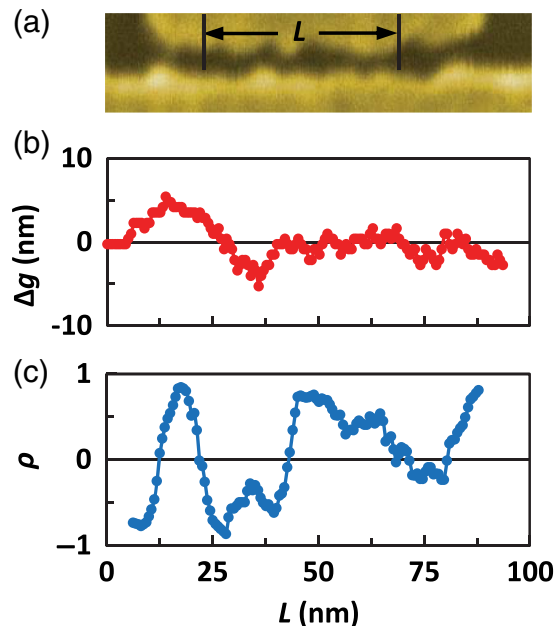
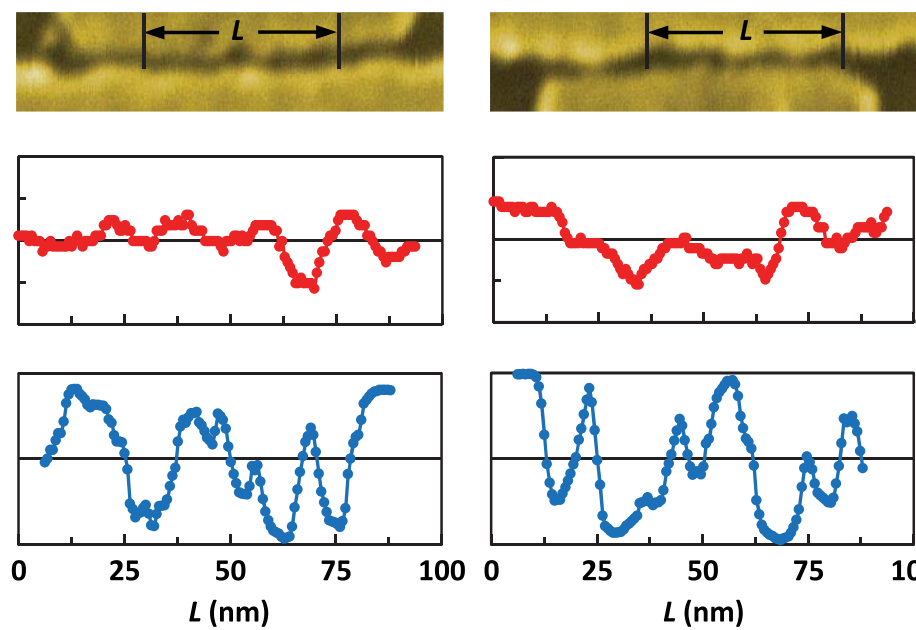

1000
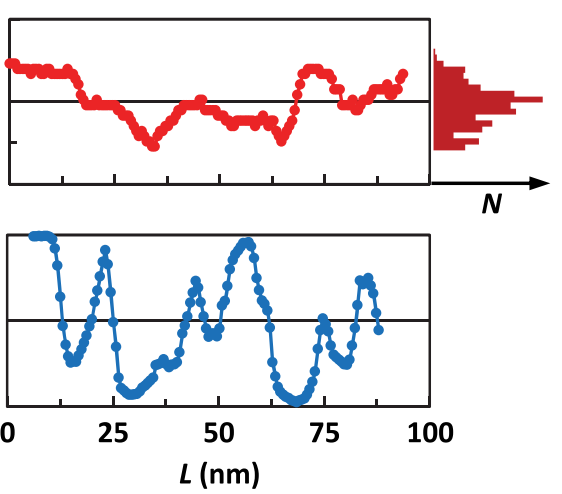

Fig. 5 Gap analysis of three high-resolution SEM images. (a) Colorized SEM images of the analyzed gaps showing the studied distance along the gap, $L$. (b) Plots of gap variation from the mean gap width values-5.92, 8.29, and $7.99 \mathrm{~nm}$ from left to right. The histogram to the right displays the relative counts for ranges of gap widths around the mean overall value, $7.4 \pm 2.2 \mathrm{~nm}$. (c) Plots of the population correlation coefficient between the two edges comprising each gap.

the primary structure. There are also, however, ranges in the same order of length over which the edges are anticorrelated. Considering the total gap lengths studied, there does not appear to be a net correlation among the edge positions across a given gap. Therefore, with the nanomasking fabrication process, an expected gap width can be patterned with a relatively high degree of accuracy, but the roughness of the gap on the secondary structure edge is not necessarily defined by the primary structure surface roughness.

This corroborates previous discussion of the gap roughness for electrical measurements, which found the secondary electrodes to be rougher than the primary ones, attributing this to the added roughness of the $\mathrm{Cr}_{x} \mathrm{O}_{y}$ film. ${ }^{74}$ The primary structure edge roughness is due to the resolution of the lithography process, the resist development, and structure evaporation. If the secondary structure edge roughness is not correlated with that of the primary structure, then the secondary evaporation and the $\mathrm{Cr}$ oxidation steps are the contributing factors of the additional roughness causing the anticorrelation. Using these measured results to incorporate accurate edge roughness into future, computational simulations will be highly valuable for precisely predicting and designing nanostructure properties. Previous work has shown that gap roughness can improve plasmonic devices, and that accurate models are keys to predict device behavior. ${ }^{77}$ This work produces better characterization of the gap width, roughness, and edge correlation, and will be helpful in optimizing nanostructures for applications, such as plasmonic nano-optics for enhanced spectroscopy. We plan to incorporate these measured structural results into computational electromagnetic and other simulations, further aiding in the fabrication design and optimization.

\section{Conclusion}

A nanomasking fabrication technique has demonstrated the capability of simultaneous fabrication of sub-10-nm slits and sublithography-resolution nanostructures. This work advances the technique by illustrating how hybrid structures can be created using different materials in each evaporation step. The width of the secondary structures can be controlled by the degree of overlap of the two lithography patterns used in the process, and the sizes of both the nanoslits and nanostructures can overcome the resolution limit of the electron beam or photolithography process used. This work advances the nanomasking method even further by introducing blanket nanomasking, where no secondary patterning step is required, as the secondary evaporation simply covers the entire sample. This has been demonstrated to successfully eliminate the secondary lithography step from the process while still producing nanogaps adjacent to multiple structures on a substrate surface. This is another aspect of the method which increases the appeal for applications requiring mass production.

An analysis of the resulting gap structure was conducted, with the correlation among gap edges studied as well. It was found that over the length of three gaps fabricated via nanomasking under ambient $\mathrm{Cr}$ oxidation conditions, the average gap width is $7.4 \pm 2.2 \mathrm{~nm}$. There was found to be no longrange edge correlation between one side of the gap and the other, but over shorter ranges (tens of nanometers), some gap regions displayed significant correlation, and others displayed significant anticorrelation. These measured values and correlation characteristics reveal parameters that can be useful for predicting nanoscale behavior, enabling more accurate modeling, design, and optimization of nanostructures.

\section{Acknowledgments}

S. J. Bauman was supported by the Doctoral Academy Fellowship through the University of Arkansas Graduate School. A. A. Darweesh has been supported by the Iraqi Ministry of Higher Education and Scientific Research.

\section{References}

1. J. Sone et al., "Nanofabrication toward sub-10 $\mathrm{nm}$ and its application to novel nanodevices," Nanotechnology 10(2), 135-141 (1999).

2. Y. Chen and A. Pépin, "Nanofabrication: conventional and nonconventional methods," Electrophoresis 22(2), 187-207 (2001). 
3. Y.-K. Choi et al., "Sublithographic nanofabrication technology for nanocatalysts and DNA chips," J. Vac. Sci. Technol. B 21(6), 29512955 (2003).

4. A. Biswas et al., "Advances in top-down and bottom-up surface nanofabrication: techniques, applications and future prospects," Adv. Colloid Interface Sci. 170(1-2), 2-27 (2012).

5. Y. Chen, "Nanofabrication by electron beam lithography and its applications: a review," Microelectron. Eng. 135, 57-72 (2015).

6. R. Chau et al., "Integrated nanoelectronics for the future," Nat. Mater. 6(11), 810-812 (2007).

7. J. Appenzeller et al., "Toward nanowire electronics," IEEE Trans. Electron Devices 55(11), 2827-2845 (2008).

8. V. Deshpande et al., "Scaling of trigate nanowire (NW) MOSFETs to sub-7 nm width: $300 \mathrm{~K}$ transition to single electron transistor," SolidState Electron. 84, 179-184 (2013).

9. S. J. Bauman, D. T. Debu, and J. B. Herzog, "Plasmonic structures fabricated via nanomasking sub-10 nm lithography technique," Proc. SPIE 9556, 95560M (2015).

10. R. Gordon, "Surface plasmon nanophotonics: a tutorial," IEEE Nanotechnol. Mag. 2(3), 12-18 (2008).

11. G. L. Hornyak et al., Introduction to Nanoscience and Nanotechnology, CRC Press, Boca Raton, Florida (2008)

12. C. A. Mack, "Fifty years of Moore's law," IEEE Trans. Semicond. Manuf. 24(2), 202-207 (2011).

13. Z.-Y. Li, "Optics and photonics at nanoscale: principles and perspectives," Europhys. Lett. 110(1), 14001 (2015).

14. N. Dean, "Colouring at the nanoscale," Nat. Nanotechnol. 10(1), 15-16 (2015).

15. J. R. Krenn et al., "Surface plasmon micro- and nano-optics," J. Microsc. 209(3), 167-172 (2003).

16. N. J. Halas, "Plasmonics: an emerging field fostered by nano letters," Nano Lett. 10(10), 3816-3822 (2010).

17. S. Enoch and N. Bonod, Plasmonics: From Basics to Advanced Topics, Springer, Berlin (2012)

18. T. J. Davis, "Plasmonics: the convergence between optics and electronics," Proc. SPIE 8923, 89232R (2013).

19. S. J. Bauman et al., "Optical nanogap matrices for plasmonic enhancement applications," Proc. SPIE 9163, 91631A (2014).

20. D. R. Ward et al., "Optical rectification and field enhancement in a plasmonic nanogap," Nat. Nanotechnol. 5(10), 732-736 (2010).

21. J. N. Krishnan et al., "Fabrication and characterization of noble metal nanowires for nanogap devices," ECS Trans. 25(41), 41-47 (2010).

22. H. C. Kim and X. Cheng, "Gap surface plasmon polaritons enhanced by a plasmonic lens," Opt. Lett. 36(16), 3082-3084 (2011).

23. A. García-Martín et al., "Field enhancement in subnanometer metallic gaps," Phys. Rev. B 83(19), 193404 (2011).

24. X. Chen et al., "Nanogap-enhanced infrared spectroscopy with template-stripped wafer-scale arrays of buried plasmonic cavities," Nano Lett. 15(1), 107-113 (2015)

25. S. J. Bauman, A. A. Darweesh, and J. B. Herzog, "Surface-enhanced Raman spectroscopy substrate fabricated via nanomasking technique for biological sensor applications," Proc. SPIE 9759, 97591I (2016).

26. S. Davies and B. Khamsehpour, "Focused ion beam machining and deposition for nanofabrication," Vacuum 47(5), 455-462 (1996).

27. B. D. Huey and R. M. Langford, "Low-dose focused ion beam nanofabrication and characterization by atomic force microscopy," Nanotechnology 14(3), 409-412 (2003).

28. O. Scholder et al., "Helium focused ion beam fabricated plasmonic antennas with sub-5 nm gaps," Nanotechnology 24(39), 395301 (2013)

29. D. R. Ward et al., "Electromigrated nanoscale gaps for surface-enhanced Raman spectroscopy," Nano Lett. 7(5), 1396-1400 (2007).

30. D. R. Ward et al., "Electronic and optical properties of electromigrated molecular junctions," J. Phys. Condens. Matter 20(37), 374118 (2008)

31. J. M. Baik, S. J. Lee, and M. Moskovits, "Polarized surface-enhanced Raman spectroscopy from molecules adsorbed in nano-gaps produced by electromigration in silver nanowires," Nano Lett. 9(2), 672-676 (2009).

32. T. Ohata et al., "Fabrication of nanogap electrode using electromigration method during metal deposition," in 7th IEEE Int. Conf. on Nano/Micro Engineered and Molecular Systems (NEMS), pp. 290-293 (2012)

33. M. Ito et al., "Simultaneous fabrication of nanogaps using field-emission-induced electromigration," in Int. Conf. on Manipulation, Manufacturing and Measurement on the Nanoscale (3M-NANO), pp. 312-315 (2014)

34. R. J. P. Keijsers et al., "Thin film mechanically controllable break junctions," Rev. Sci. Instrum. 67(8), 2863-2866 (1996).

35. A. A. Tseng, "Recent developments in nanofabrication using focused ion beams," Small 1(10), 924-939 (2005).

36. F. Watt et al., "Ion beam lithography and nanofabrication: a review," Int. J. Nanosci. 4(3), 269-286 (2005).

37. G. M. Whitesides and B. Grzybowski, "Self-assembly at all scales," Science 295(5564), 2418-2421 (2002).

38. J. Zhang et al., "Colloidal self-assembly meets nanofabrication: from two-dimensional colloidal crystals to nanostructure arrays," $A d v$. Mater. 22(38), 4249-4269 (2010).
39. J. Chen et al., "One-step fabrication of sub-10-nm plasmonic nanogaps for reliable SERS sensing of microorganisms," Biosens. Bioelectron. 44 191-197 (2013)

40. J. Chen et al., "Fabrication of tunable Au SERS nanostructures by a versatile technique and application in detecting sodium cyclamate," RSC Adv. 4(43), 22660-22668 (2014)

41. J. Fontana and B. R. Ratna, "Toward high throughput optical metamaterial assemblies," Appl. Opt. 54(31), F61-F69 (2015).

42. H. Cai et al., "Wafer scale fabrication of highly dense and uniform array of sub-5 nm nanogaps for surface enhanced Raman scatting substrates," Opt. Express 24(18), 20808-20815 (2016).

43. J. Fontana et al., "Linear and nonlinear optical characterization of selfassembled, large-area gold nanosphere metasurfaces with sub-nanometer gaps," Opt. Express 24(24), 27360-27370 (2016).

44. P. Pandey et al., "Nanoparticles to nanoholes: fabrication of porous $\mathrm{GaN}$ with precisely controlled dimension via the enhanced $\mathrm{GaN}$ decomposition by Au nanoparticles," Cryst. Growth Des. 17(6), 3334-3344 (2016).

45. S.-W. Lee et al., "Suppression of resist contamination during photolithography on carbon nanomaterials by a sacrificial layer," Carbon 66(Suppl. C), 295-301 (2014)

46. H. K. Raut et al., "Multiscale ommatidial arrays with broadband and omnidirectional antireflection and antifogging properties by sacrificial layer mediated nanoimprinting," ACS Nano 9(2), 1305-1314 (2015).

47. C. A. Joiner et al., "Cleaning graphene with a titanium sacrificial layer," Appl. Phys. Lett. 104(22), 223109 (2014).

48. P. Xiao et al., "A sacrificial-layer approach to fabricate polysulfone support for forward osmosis thin-film composite membranes with reduced internal concentration polarisation,” J. Membr. Sci. 481(Suppl. C), 106-114 (2015)

49. Y. Wei et al., "Screen printing of a capacitive cantilever-based motion sensor on fabric using a novel sacrificial layer process for smart fabric applications," Meas. Sci. Technol. 24(7), 075104 (2013).

50. Y. Miyamoto et al., "Preparation of self-supporting Au thin films on perforated substrate by releasing from water-soluble sacrificial layer," Jpn. J. Appl. Phys. 55(7S2), 07LE05 (2016).

51. A. Kannegulla and L.-J. Cheng, "Metal assisted focused-ion beam nanopatterning," Nanotechnology 27(36), 36LT01 (2016).

52. S. J. Bauman et al., "Fabrication of sub-lithography-limited structures via nanomasking technique for plasmonic enhancement applications," IEEE Trans. Nanotechnol. 14(5), 790-793 (2015).

53. J. N. Anker et al., "Biosensing with plasmonic nanosensors," Nat. Mater. 7(6), 442-453 (2008).

54. S. Kemme, Microoptics and Nanooptics Fabrication, CRC Press, Boca Raton, Florida (2009).

55. G. Baffou and R. Quidant, "Thermo-plasmonics: using metallic nanostructures as nano-sources of heat," Laser Photonics Rev. 7(2), 171-187 (2013)

56. S. Ahn, D. Rourke, and W. Park, "Plasmonic nanostructures for organic photovoltaic devices," J. Opt. 18(3), 033001 (2016)

57. H. Guo and J. Guo, "Hybrid plasmon photonic crystal resonance grating for integrated spectrometer biosensor," Opt. Lett. 40(2), 249-252 (2015).

58. T. Lu et al., "Bio-inspired fabrication of stimuli-responsive photonic crystals with hierarchical structures and their applications," Nanotechnology 27(12), 122001 (2016).

59. W. Lu and C. M. Lieber, "Nanoelectronics from the bottom up," Nat. Mater. 6(11), 841-850 (2007).

60. C. Clavero, "Plasmon-induced hot-electron generation at nanoparticle/ metal-oxide interfaces for photovoltaic and photocatalytic devices," Nat. Photonics 8(2), 95-103 (2014).

61. S. M. Iqbal et al., "Direct current electrical characterization of ds-DNA in nanogap junctions," Appl. Phys. Lett. 86(15), 153901 (2005).

62. A. Pallaoro et al., "Combined surface-enhanced Raman spectroscopy biotags and microfluidic platform for quantitative ratiometric discrimination between noncancerous and cancerous cells in flow," $J$ Nanophotonics 7(1), 073092 (2013).

63. A. I. Nusir and M. O. Manasreh, "Self-powered near-infrared photodetector based on asymmetrical Schottky interdigital contacts," IEEE Electron Device Lett. 36(11), 1172-1175 (2015).

64. T. Siegfried et al., "Engineering metal adhesion layers that do not deteriorate plasmon resonances," ACS Nano 7(3), 2751-2757 (2013).

65. W. Chang et al., "Tuning the acoustic frequency of a gold nanodisk through its adhesion layer," Nat. Commun. 6, 7022 (2015).

66. F. Colas et al., "Comparison of adhesion layers of gold on silicate glasses for SERS detection," J. Opt. 17(11), 114010 (2015).

67. A. I. Nusir et al., "Hot electrons in microscale thin-film Schottky barriers for enhancing near-infrared detection," IEEE Photonics Technol. Lett. 28(20), 2241-2244 (2016).

68. D. T. Debu et al., "Surface plasmon damping effects due to Ti adhesion layer in individual gold nanodisks," Opt. Mater. Express 7(1), 73-84 (2017).

69. M. Akbari et al., "Influence of Au thickness on the performance of plasmonic enhanced hematite photoanodes," RSC Adv. 3(39), 1783717842 (2013) 
70. A. Dhar, Z. Zhao, and T. L. Alford, "Effect of gold thickness and annealing on optical and electrical properties of $\mathrm{TiO}_{2} / \mathrm{Au} / \mathrm{TiO}_{2}$ multilayers as transparent composite electrode on flexible substrate," JOM 67(4), 840-844 (2015).

71. A. Bhukta et al., "Effect of Au thickness on AuAg bimetallic growth on reconstructed Si(5 5 12) surfaces," Appl. Phys. A 123(3), 174 (2017)

72. J. R. Dunklin et al., "Plasmonic extinction in gold nanoparticle-polymer films as film thickness and nanoparticle separation decrease below resonant wavelength," J. Nanophotonics 11(1), 016002 (2017).

73. P. K. Ghosh et al., "Calculated thickness dependent plasmonic properties of gold nanobars in the visible to near-infrared light regime," PLoS One 12(5), e0177463 (2017).

74. A. Fursina et al., "Nanogaps with very large aspect ratios for electrical measurements," Appl. Phys. Lett. 92(11), 113102 (2008).

75. A. A. Darweesh, S. J. Bauman, and J. B. Herzog, "Improved optical enhancement using double-width plasmonic gratings with nanogaps," Photonics Res. 4(5), 173-180 (2016).

76. S. J. Bauman et al., "Substrate oxide layer thickness optimization for a dual-width plasmonic grating for surface-enhanced Raman spectroscopy (SERS) biosensor applications," Sensors 17(7), 1530 (2017).

77. J. B. Herzog et al., "Dark plasmons in hot spot generation and polarization in interelectrode nanoscale junctions," Nano Lett. 13(3), 13591364 (2013).

Stephen J. Bauman is a doctoral candidate in the Microelectronics and Photonics Graduate Program, the University of Arkansas. He received his BS degree from Southeast Missouri State University and his MS degree from the University of Arkansas. He is an SPIE member as well as a cofounder and chapter officer of the Arkansas Laserbacks SPIE student chapter at the University of Arkansas.

Ahmad A. Darweesh is a doctoral candidate in the Microelectronics and Photonics Graduate Program, the University of Arkansas. He received his $\mathrm{BSc}$ and $\mathrm{MSc}$ degrees from Almostansiriyah University in 1999 and 2001, respectively. He was supported by the Iraqi Ministry of Higher Education and Scientific Research. His research in nanooptics now focuses on plasmonic nanostructures.

Desalegn T. Debu is a doctoral candidate in the Department of Physics, the University of Arkansas. He received his MS degree in physics from Addis Ababa University, Ethiopia, in 2007 and his BS degree in physics from Dilla University, Ethiopia, in 2004.

Joseph B. Herzog is an assistant professor in the Department of Physics, the University of Arkansas. He received his PhD from the University of Notre Dame, and then was a postdoc at Rice University. He was a 2017 Fellow at the U.S. Naval Research Laboratory in Washington, DC. His work experience includes Intel Corporation and the BESSY synchrotron in Berlin, Germany. His research in nanooptics now focuses on plasmonic nanostructures. 